\title{
Quantum Interference at the Twist Boundary in Graphene
}

\author{
S. Shallcross, ${ }^{1, *}$ S. Sharma, ${ }^{2,3}$ and O. A. Pankratov ${ }^{1}$ \\ ${ }^{1}$ Lehrstuhl für Theoretische Festkörperphysik, Staudstrasse 7-B2, 91058 Erlangen, Germany \\ ${ }^{2}$ Fritz Haber Institute of the Max Planck Society, Faradayweg 4-6, D-14195 Berlin-Dahlem, Germany \\ ${ }^{3}$ Institut für Theoretische Physik, Freie Universität Berlin, Arnimallee 14, D-14195 Berlin, Germany
}

(Received 15 April 2008; published 1 August 2008)

\begin{abstract}
We explore the consequences of a rotation between graphene layers for the electronic spectrum. We derive the commensuration condition in real space and show that the interlayer electronic coupling is governed by an equivalent commensuration in reciprocal space. The larger the commensuration cell, the weaker the interlayer coupling, with exact decoupling for incommensurate rotations and in the $\theta \rightarrow 0$ limit. Furthermore, from first-principles calculations we determine that even for the smallest possible commensuration cell the decoupling is effectively perfect, and thus graphene layers will be seen to decouple for all rotation angles.
\end{abstract}

DOI: 10.1103/PhysRevLett.101.056803

Uniquely in condensed matter physics, graphene, a two-dimensional crystal of carbon, offers a realization of the massless Dirac equation. This situation arises from the symmetry of the graphene honeycomb lattice that, for low energy excitations, results in a linear energy-momentum dispersion and an internal degree of freedom similar to that of chirality, features which mimic those of massless Dirac fermions. From this follows a wealth of novel electronic properties including an anomalous phase in the integer quantum Hall effect, $\sqrt{B}$ dependence of Landau level energies, and suppressed backscattering leading to quasiballistic transport [1,2].

Rich and novel physics also arises in stacks of two or more atomic layers of graphene, so-called few-layer graphene (FLG) systems. In particular, the graphene bilayer has received a great deal of attention [3]. Here the electronic excitations are, as in the case of single-layer graphene (SLG), chiral, but the electronic coupling of the layers results in a quadratic energy-momentum dispersion; they behave as massive Dirac quasiparticles. This system is particularly interesting for possible future applications as a controllable gap may be opened with an external electric field, as recently realized for the case of exfoliated graphene on a $\mathrm{SiO}_{2}$ substrate [4].

The structural degrees of freedom of FLG are the translations and rotations of the constituent layers. While the electronic consequences of the former degree of freedom have been explored $[5,6]$, only very recently has the rotational degree of freedom received attention. This has been stimulated by the observation of Hass et al. [7] that graphene grown epitaxially on $\mathrm{SiC}(000 \overline{1})$ contains a high density of twist boundary faults, i.e., layers with a relative rotation. The same authors demonstrated that a bilayer structure with the relative rotation frequently observed in experiment $\left(\theta=30^{\circ} \pm 2.20^{\circ}\right)$ manifests an apparent electronic decoupling. Namely, ab initio calculations showed that both layers displayed the Dirac cone and Fermi velocity characteristic of SLG [7]. This remarkable result has inspired a number of subsequent theoretical and experi-
PACS numbers: 73.21.Ac, 81.05.Uw

mental works [8-10]. In Ref. [8], bilayer and trilayer twist boundary systems were investigated by ab initio calculations, and in both cases layers with a relative rotation were found to display apparent SLG behavior. On the other hand, Ref. [9] considered the $\theta \rightarrow 0$ limit of the rotated bilayer in a continuum approximation, with the result that the layers exactly decoupled but with the Fermi velocity of the Dirac cone suppressed as compared to SLG. This latter result is in striking contrast to ab initio calculations $[7,8]$ for finite rotation angles where a Fermi velocity exactly equal to that of SLG is found.

Since to date only specific rotation angles or limits have been investigated, an outstanding and fundamental question is the identity of the full set of rotation angles that cause decoupling. Of equal interest is the basic physical mechanism underlying this decoupling which, at this stage, remains unclear. In this work we demonstrate that all finite rotation angles that give rise to commensurate structures lead to approximate, although for nearly all cases effectively perfect, decoupling with exact decoupling only in the limit $\theta \rightarrow 0$ or for incommensurate rotations. The reason for this remarkable behavior we identify as a destructive interference between the layers, which may be expressed by a commensuration condition in reciprocal space.

Before investigating the rotated bilayer on the electronic level, it is essential to understand the crystallographic problem posed; i.e., to find the set of rotation angles which give rise to commensurate crystal structures. It is easy to see that, while the resulting structure will depend on the initial stacking and choice of rotation axis, the existence of a commensuration depends only on the mutual orientation of the primitive lattice vectors of each layer. The problem is thus reduced to finding rotations such that applied to the parent triangular lattice leave a subset of atoms coincident with the original unrotated lattice. This condition may be expressed as $\mathbf{a}_{2}=\mathbf{R} \mathbf{a}_{1}$, with $\mathbf{a}_{1,2}$ direct-lattice vectors and $\mathbf{R}$ the rotation operator. In natural lattice coordinates, this condition reads 


$$
\left(\begin{array}{l}
m_{1} \\
m_{2}
\end{array}\right)=\mathbf{T}^{-1} \mathbf{R} \mathbf{T}\left(\begin{array}{l}
n_{1} \\
n_{2}
\end{array}\right),
$$

where $\mathbf{T}$ is the operator that transforms between the triangular lattice and Cartesian coordinate systems. Both $\mathbf{n}$ and $\mathbf{m}$, being expressed in the lattice coordinate system, are integer-valued, and hence Eq. (1) is a Diophantine system which, with a standard choice of basis vectors, may be written as

$$
\left(\begin{array}{l}
m_{1} \\
m_{2}
\end{array}\right)=\left(\begin{array}{cc}
\frac{1}{\sqrt{3}} \sin \theta+\cos \theta & \frac{2}{\sqrt{3}} \sin \theta \\
-\frac{2}{\sqrt{3}} \sin \theta & -\frac{1}{\sqrt{3}} \sin \theta+\cos \theta
\end{array}\right)\left(\begin{array}{l}
n_{1} \\
n_{2}
\end{array}\right) .
$$

A necessary condition for an integer solution of this equation is that the matrix be rational-valued which implies that $\cos \theta=i_{2} / i_{3}$ and $\sin \theta / \sqrt{3}=i_{1} / i_{3}$ and so $3 i_{1}^{2}+i_{2}^{2}=i_{3}^{2}$, where $\left\{i_{1}, i_{2}, i_{3}\right\} \in \mathbb{Z}$. Solution of this Diophantine equation yields

$$
\begin{gathered}
i_{1}=p^{2}-2 p q-3 q^{2}, \\
i_{2}=-\left(p^{2}+6 p q-3 q^{2}\right), \\
i_{3}=2\left(p^{2}+3 q^{2}\right),
\end{gathered}
$$

where $p, q \in \mathbb{Z}$. Substitution back into Eq. (2) then yields

$$
\begin{aligned}
& i_{3} m_{1}=\left(i_{2}+i_{1}\right) n_{1}+\left(2 i_{1}\right) n_{2}, \\
& i_{3} m_{2}=\left(-2 i_{1}\right) n_{1}+\left(i_{2}-i_{1}\right) n_{2},
\end{aligned}
$$

and so the problem is reduced to that of simultaneous Diophantine equations for the unknown integers $n_{1}, n_{2}$, $m_{1}$, and $m_{2}$.

One may show that for any $(p, q)$ pair these equations have infinitely many solutions which correspond to two superlattices of sites related by Eq. (1). Thus every commensuration of two graphene layers may be labeled by a $(p, q)$ integer pair. What remains is to determine, from these superlattices, the primitive commensuration cells. Defining $\delta=3 / \operatorname{gcd}(p, 3)$, we find for $\delta=1$

$$
\begin{aligned}
\mathbf{t}_{1} & =\frac{1}{\gamma}(p+3 q) \mathbf{a}_{1}+\frac{1}{\gamma}(p-3 q) \mathbf{a}_{2}, \\
\mathbf{t}_{2} & =-\frac{1}{\gamma}(2 p) \mathbf{a}_{1}+\frac{1}{\gamma}(p+3 q) \mathbf{a}_{2},
\end{aligned}
$$

and for $\delta=3$

$$
\begin{aligned}
& \mathbf{t}_{1}=\frac{1}{\gamma}(p+q) \mathbf{a}_{1}-\frac{1}{\gamma}(2 q) \mathbf{a}_{2}, \\
& \mathbf{t}_{2}=-\frac{1}{\gamma}(p-q) \mathbf{a}_{1}+\frac{1}{\gamma}(p+q) \mathbf{a}_{2},
\end{aligned}
$$

where $\gamma=\operatorname{gcd}(p+3 q, p-3 q)$. [Examples of such commensuration cells are shown in the lower panel inset in Fig. 2. The left inset corresponds to $(p, q)=(1,3)$, with $(p, q)=(3,5)$ shown on the right.] The commensurations found in Ref. [9] are a special case of those presented here; they may be recovered by the choice $(p, q)=(1,2 i+1)$ for which always $\gamma=2$ and $\delta=3$. From these primitive vectors may then be found a more convenient definition of the rotation angle

$$
\theta=\cos ^{-1}\left(\frac{3 q^{2}-p^{2}}{3 q^{2}+p^{2}}\right)
$$

(it is defined such that for $p \geq q$ we have $0<\theta<\pi / 3$ ), and the number of primitive vectors from each layer in the commensuration cell

$$
N=\frac{3}{\delta} \frac{1}{\gamma^{2}}\left(3 q^{2}+p^{2}\right),
$$

with the number of carbon atoms in the cell equal to $4 N$. This factor of 4 is simply due to the fact there are two layers in the cell and two basis atoms in the honeycomb structure. The rapidly increasing sequence described by Eq. (10) as $\theta \rightarrow 0\left[4 N=\left(\sin ^{2} \theta / 2\right)^{-1}\right.$ is the lower bound] makes $a b$ initio calculations for the full range of angles rather prohibitive. Fortunately, as we shall now show, a general statement on the angular dependence of the electronic coupling is possible.

We consider two graphene layers with the second rotated relative to the first. Because of the different translational symmetries of each layer, it is natural to decompose the bilayer potential as $V^{(1)}+V^{(2)}+\delta V$. The potentials $V^{(1,2)}$ have single-layer translation symmetry; $\mathbf{T}_{\mathbf{a}} V^{(1)}=V^{(1)}$ and $\mathbf{T}_{\mathbf{R a}} V^{(2)}=V^{(2)}$, while $\delta V$ has only the translational symmetry of the bilayer as a whole. Since the layers are weakly interacting, we shall assume that $\delta V$ may be neglected; possible consequences of this potential will be investigated in numerical simulations.

A complete basis for the bilayer is that formed from the eigenkets of the single-layer problems, i.e., from $\left\{\left|\phi_{i \mathbf{k}}^{(n)}\right\rangle\right\}$ with $\mathbf{H}^{(n)}\left|\phi_{i \mathbf{k}}^{(n)}\right\rangle=\left(\frac{\mathbf{p}^{2}}{2 m}+\mathbf{V}^{(n)}\right)\left|\phi_{i \mathbf{k}}^{(n)}\right\rangle=\epsilon_{i \mathbf{k}}^{(n)}\left|\phi_{i \mathbf{k}}^{(n)}\right\rangle$. Here $n$ is the layer index, $\mathbf{k}$ a $\mathbf{k}$-vector index, and $i$ a state index. The Hamiltonian of the bilayer is diagonal in the $\mathbf{k}$ index only for $\mathbf{k}$ vectors in the corresponding Brillouin zone (BZ), and so to use this fact the basis elements must be folded back to this superlattice BZ. This procedure amounts to renumbering the basis elements between the $i$ and $\mathbf{k}$ indices. Expressing the Hamiltonian of the rotated bilayer $\mathbf{H}=$ $\frac{\mathbf{p}^{2}}{2 m}+\mathbf{V}^{(1)}+\mathbf{V}^{(2)}$ in this basis as

$$
\begin{aligned}
{[\mathbf{H}(\mathbf{k})]_{i j}=} & \left(\begin{array}{cc}
\epsilon_{i \mathbf{k}}^{(1)} \delta_{i j} & 0 \\
0 & \epsilon_{i \mathbf{k}}^{(2)} \delta_{i j}
\end{array}\right) \\
& +\left(\begin{array}{cc}
\left\langle\phi_{i \mathbf{k}}^{(1)}\left|\mathbf{V}^{(2)}\right| \phi_{i \mathbf{k}}^{(1)}\right\rangle & \left\langle\phi_{i \mathbf{k}}^{(1)}|\mathbf{H}| \phi_{j \mathbf{k}}^{(2)}\right\rangle \\
\left\langle\phi_{j \mathbf{k}}^{(2)}|\mathbf{H}| \phi_{i \mathbf{k}}^{(1)}\right\rangle & \left\langle\phi_{j \mathbf{k}}^{(2)}\left|\mathbf{V}^{(1)}\right| \phi_{j \mathbf{k}}^{(2)}\right\rangle
\end{array}\right),
\end{aligned}
$$

we see that the term causing interlayer hybridization involves always an overlap between basis functions or operators of different translational symmetry. Using the plane wave expansions $\left\langle\mathbf{r}\left|\mathbf{V}^{(2)}\right| \mathbf{r}^{\prime}\right\rangle=\sum_{\mathbf{R G}} V_{\mathbf{R G}}^{(2)} e^{i \mathbf{R G} \cdot \mathbf{r}} \delta(\mathbf{r}-$ $\left.\mathbf{r}^{\prime}\right)$ and $\left\langle\mathbf{r} \mid \phi_{j \mathbf{k}}^{(1)}\right\rangle=(1 / \sqrt{V}) \sum_{\mathbf{G}} c_{j \mathbf{k}+\mathbf{G}} e^{i(\mathbf{k}+\mathbf{G}) \cdot \mathbf{r}}$ one finds that 
overlap integrals of the type $\left\langle\phi_{i \mathbf{k}}^{(1)}\left|\mathbf{V}^{(2)}\right| \phi_{j \mathbf{k}}^{(1)}\right\rangle$ have nonzero terms in such an expansion only where $\left(\mathbf{k}_{1}-\mathbf{k}_{2}\right)+\mathbf{G}_{1}-$ $\mathbf{G}_{2}-\mathbf{R G}_{3}=\mathbf{0}$. Similar results are found for the other terms; e.g., for the term $\left\langle\phi_{i \mathbf{k}}^{(1)}|\mathbf{H}| \phi_{j \mathbf{k}}^{(2)}\right\rangle$, we find $\left(\mathbf{k}_{1}-\right.$ $\left.\mathbf{k}_{2}\right)+\mathbf{G}_{1}-\mathbf{R G}_{2}=\mathbf{0}, \quad\left(\mathbf{k}_{1}-\mathbf{k}_{2}\right)+\mathbf{G}_{1}-\mathbf{G}_{2}-\mathbf{R G}_{3}=$ $\mathbf{0}$, and $\left(\mathbf{k}_{1}-\mathbf{k}_{2}\right)+\mathbf{G}_{1}-\mathbf{R}\left(\mathbf{G}_{2}+\mathbf{G}_{3}\right)=\mathbf{0}$ for, respectively, the kinetic energy and $\mathbf{V}^{(1)}$ and $\mathbf{V}^{(2)}$ operators (where $\mathbf{k}_{1}$ and $\mathbf{k}_{2}$ are $\mathbf{k}$ vectors of plane waves before backfolding to the commensuration Brillouin zone).

All of these are commensuration conditions for the reciprocal lattices of the two layers which, since the reciprocal lattice is also hexagonal, correspond exactly to the conditions for determining crystal commensuration in real space. A fraction $1 / N^{2}$ of the terms in the expansions of all overlap integrals will therefore be identically zero. However, due to the backfolding procedure of the basis functions, the size of the Hamiltonian matrix increases also by $N^{2}$, and so this reduction of the number of terms does not prove the vanishing of interlayer hybridization. The crucial point is that the expansion coefficients approach zero as $|\mathbf{G}| \rightarrow \infty$, and so the commensuration conditions ensure coupling only through $\mathbf{G}$ vectors of very low weight, and therefore the interlayer hybridization becomes weaker as $N$ increases. Essentially, the rotation of graphene layers causes a destructive interference which reduces interlayer hybridization. On the other hand, for the unrotated $A B$ stacked bilayer, $\mathbf{R} \equiv \mathbf{1}$ and the interaction is through all $\mathbf{G}$ vectors, sufficient to cause a splitting of $\approx 0.8 \mathrm{eV}$ between bonding and antibonding states at the $\mathbf{K}$ point and the destruction of the linear spectrum. This analysis therefore predicts that there may be some residual interlayer hybridization for small $N$, and hence splitting of bands at the $\mathbf{K}$ point and nonlinear behavior, but as $N \rightarrow \infty$ this will vanish. To test this prediction, and to explore the possible role of the neglected $\delta \mathbf{V}$ term, we now turn to first-principles simulations of the rotated graphene bilayer.

Our calculations have been performed using the local density approximation (LDA) to density functional theory, with the Kohn-Sham equations solved within the projected augmented wave method [11], as implemented in the electronic structure program VASP $[12,13]$. We have crosschecked several results with the full potential all electron code EXCITING [14]. It is well known that the LDA fails to describe dispersion forces and that these are important for graphite and, presumably, also for few-layer graphene systems. On the other hand, numerous studies have confirmed that the LDA does provide a good description of the band structure of graphite and graphene systems [7,15,16], and so it is sufficient for our purposes here. Characterization of a general bilayer requires that the initial stacking, choice of rotation axis, and rotation angle be specified. For conciseness of expression, we adopt a nomenclature whereby we first specify the initial stacking $(A A$ or $A B)$, then the choice of rotation axis - assumed a high symmetry point - by the number of $\mathrm{C}$ atoms it intersects $(-0 a$,
$-1 a,-2 a)$, and finally the rotation angle, e.g., $(A B, 2 a)$ $\theta=38.21^{\circ}$.

In Fig. 1 is displayed the dependence of the $\mathbf{K}$ point splitting on $N$ for commensuration cells of the $(A B, 2 a)$ type. Although there is a splitting of $\approx 7 \mathrm{meV}$ for the lowest commensuration cell $(N=7)$, this rapidly decreases as $N$ increases, a result we found to be robust against all numerical parameters, e.g., the vacuum separation of the bilayer slabs. The graphene bilayer therefore effectively decouples for all rotation angles.

As may be seen in the upper panel in Fig. 2, the band structure of the graphene bilayer [the case $(A B, 2 a) N=7$ is shown] is not identical to that of SLG away from the $\mathbf{K}$ point. To determine the origin of this, we have calculated the $N=7 A B A$ trilayer with the rotation applied to the middle layer. In this case (lower panel in Fig. 2) there is always one band which falls nearly exactly on that of single-layer graphene. The main difference between these bilayer and trilayer systems is that in the former the graphene planes are in an unsymmetrical environment, quite different from that of single-layer graphene, while in the latter this symmetry is restored for the middle plane. The differences between the bilayer and single-layer band structure are thus not due to a covalent interaction between the layers, which would not be affected by global symmetry, but instead due to electrostatic effects arising from the lower symmetry of the bilayer.

We now consider the impact of the crystal structure of the rotated bilayer. Shown in the upper panel in Fig. 2 are, in fact, in addition to the band structures of the $(A B, 2 a)$ $\theta=21.79^{\circ}$ bilayer (crystal structure shown in top left inset) and $(A B, 2 a) \theta=38.21^{\circ}$ bilayer (top right inset), seven bilayers formed by taking the $(A A, 0 a) \theta=38.21^{\circ}$ cell and applying a translation $\delta \mathbf{a}=\eta\left(\mathbf{a}_{1}+\mathbf{a}_{2}\right)$ to one layer (lower inset for $\eta=0.1$ ). Remarkably, the band

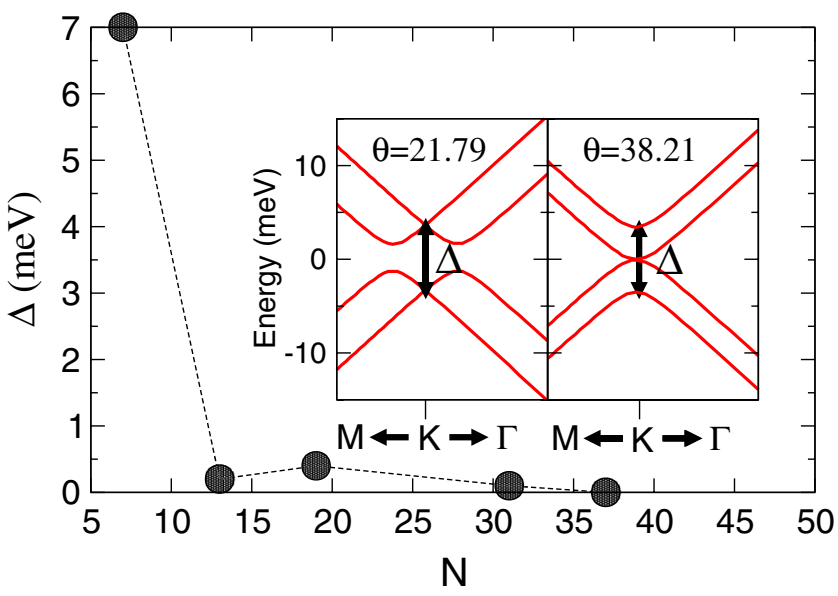

FIG. 1 (color online). The inset graph displays band structure near the Dirac point for the $(A B, 2 a) \theta=30^{\circ} \pm 8.21^{\circ}$ commensuration cells $(N=7)$. Clearly, near the Dirac point the $\pi$ and $\pi^{*}$ bands are not linear. The main graph details the rapid decay, as $N$ increases, of the energy window where such deviations from linearity occur. 


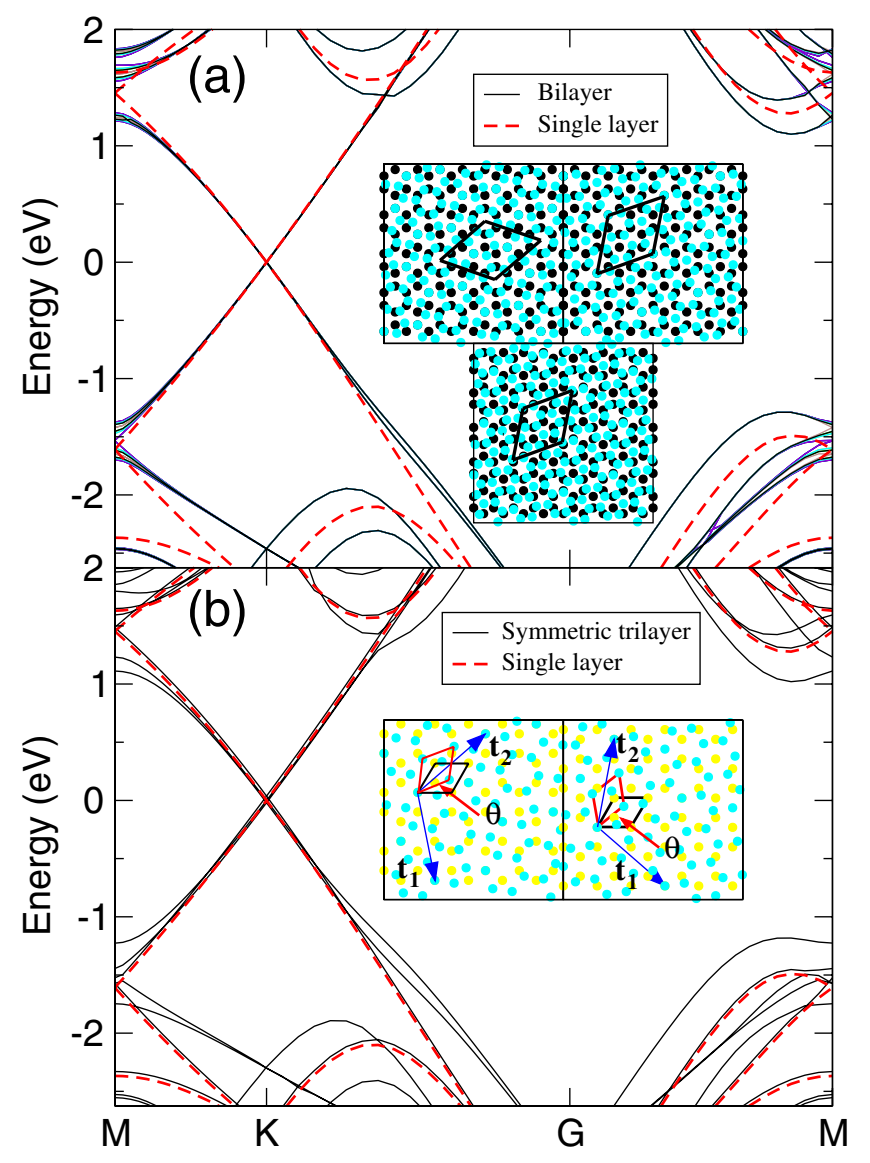

FIG. 2 (color online). (a) Band structure of several rotated bilayer structures for $\theta=30^{\circ} \pm 8.21^{\circ}$ (corresponding to $N=$ 7 ); the inset shows resulting atomic structures where shading distinguishes atoms belonging to different layers. (b) Band structure of the single-layer graphene and the $A B A$ trilayer with the middle layer rotated by $\theta=21.79^{\circ}$. The inset displays close-ups of structures in (a) with commensuration primitive vectors [Eqs. (7) and (8)] and rotation angle [Eq. (9)] shown.

structure is almost independent of these changes in crystal structure, the largest difference being found at the $\mathbf{M}$ point. This indicates that the $\delta \mathbf{V}$ contribution to the bilayer potential, which would depend on details of the crystal structure, is not important. Complementary to this, we find that the sliding energy of the rotated bilayer is less than $10^{-6} \mathrm{eV}$, a result already noted in Ref. [15].

We now turn to the question of the Fermi velocity $v_{F}$ at the $\mathbf{K}$ point of the rotated bilayer. It is clear from Fig. 2 that our calculations show $v_{F}$ of the bilayer to be equal to that of SLG, a feature that we find for all rotation angles we have studied $\left(\theta=30^{\circ} \pm 2.20^{\circ}, 30^{\circ} \pm 8.21^{\circ}, 30^{\circ} \pm\right.$ $12.10^{\circ}$, and $30^{\circ} \pm 20.57^{\circ}$ ), in agreement with all other ab initio studies $[7,8]$. This, however, is in contrast to the finding of Ref. [9], where, by deploying a continuum approximation, the small angle limit was investigated and $v_{F}$ found to be suppressed. Experimentally, the evidence for this seems uncertain as a suppression of $v_{F}$ is found for graphene stacks grown on both the $\mathrm{C}$ and $\mathrm{Si}$ faces of $\mathrm{SiC}$, whereas twist boundary faults appear only during growth on the $\mathrm{C}$ face [17]. This disagreement between the continuum approximation for the (singular) $\theta \rightarrow 0$ limit and real lattice calculations requires further investigation but is beyond the scope of the present work.

Finally, we note that the rotation of one layer in an otherwise ordered graphene stack is a trivial extension of the analysis above for the rotated bilayer. The meaning of the potential operators $\mathbf{V}^{(1)}$ and $\mathbf{V}^{(2)}$ simply becomes that of the graphene stack and rotated layer, respectively, and the remainder of the analysis follows as before. The wellknown rotation of graphite surface layers leading to móire patterns is a case covered by this extension, as well as the trilayer systems studied here and in Ref. [8].

While there are currently only two experimental observations linking twist boundary faults with SLG behavior $[7,10]$, we hope the general nature of the decoupling presented stimulates the search for new situations in which effective SLG may be fabricated.

The authors acknowledge Deutsche Forschungsgemeinschaft for financial support and gratefully recognize collaboration within the Interdisciplinary Centre for Molecular Materials.

*sam_shallcross@yahoo.co.uk

[1] A. K. Geim and K. S. Novoselov, Nat. Mater. 6, 183 (2007).

[2] A.H. Castro Neto, F. Guinea, N.M.R. Peres, K.S. Novoselov, and A. K. Geim, arxiv:0709.1163.v1.

[3] Edward McCann, David S. L. Abergel, and Vladimir I. Fal'ko, Solid State Commun. 143, 110 (2007).

[4] Eduardo V. Castro et al., Phys. Rev. Lett. 99, 216802 (2007).

[5] Sylvain Latil and Luc Henrard, Phys. Rev. Lett. 97, 036803 (2006).

[6] Masato Aoki and Hiroshi Amawashi, Solid State Commun. 142, 123 (2007).

[7] J. Hass et al., Phys. Rev. Lett. 100, 125504 (2008).

[8] Sylvain Latil, Vincent Meunier, and Luc Henrard, Phys. Rev. B 76, 201402(R) (2007).

[9] J.M. B. Lopes dos Santos, N. M. R. Peres, and A.H. Castro Neto, Phys. Rev. Lett. 99, 256802 (2007).

[10] Francois Varchon, Pierre Mallet, Laurence Magaud, and Jean-Yves Veuillen, Phys. Rev. B 77, 165415 (2008).

[11] P. E. Blöchl, Phys. Rev. B 50, 17953 (1994).

[12] G. Kresse and J. Haffner, J. Phys. Condens. Matter 6, 8245 (1994).

[13] We use $E_{\text {cut }}=500 \mathrm{eV}$ and a $\mathbf{k}$ mesh of $12 \times 12$ for the 28 atom cell and $3 \times 3$ for the largest 148 atom cell. The interlayer spacing was set to 6.33 a.u.; displacement of $\mathrm{C}$ atoms from the equlibrium interlayer spacing was found to be negligible.

[14] J. K. Dewhurst, S. Sharma, and C. Ambrosch-Draxl, http:// exciting.sourceforge.net, 2004.

[15] Aleksey N. Kolmogorov and Vincent H. Crespi, Phys. Rev. B 71, 235415 (2005).

[16] Alexander Mattausch and Oleg Pankratov, Phys. Rev. Lett. 99, 076802 (2007).

[17] Walt A. de Heer et al., Solid State Commun. 143, 92 (2007). 